\title{
Performance Analysis of a Diesel Cycle under the Restriction of Maximum Cycle Temperature with Considerations of Heat Loss, Friction, and Variable Specific Heats
}

\author{
S.S. HOU ${ }^{a * *}$ AND J.C. $\operatorname{LIN}^{b}$ \\ ${ }^{a}$ Department of Mechanical Engineering, Kun Shan University, Tainan City 71003, Taiwan, ROC \\ ${ }^{b}$ Department of General Education, Transworld University, Touliu City, Yunlin County 640, Taiwan, ROC
}

(Received November 11, 2010)

\begin{abstract}
The objective of this study is to examine the influences of heat loss characterized by a percentage of fuel's energy, friction and variable specific heats of working fluid on the performance of an air standard Diesel cycle with the restriction of maximum cycle temperature. A more realistic and precise relationship between the fuel's chemical energy and the heat leakage that is constituted on a pair of inequalities is derived through the resulting temperature. The variations in power output and thermal efficiency with compression ratio, and the relations between the power output and the thermal efficiency of the cycle are presented. The results show that the power output as well as the efficiency where maximum power output occurs will increase with the increase of maximum cycle temperature. The temperature-dependent specific heats of working fluid have a significant influence on the performance. The power output and the working range of the cycle increase while the efficiency decreases with increasing specific heats of working fluid. The friction loss has a negative effect on the performance. Therefore, the power output and efficiency of the cycle decrease with increasing friction loss. It is noteworthy that the effects of heat loss characterized by a percentage of fuel's energy, friction and variable specific heats of working fluid on the performance of a Diesel-cycle engine are significant and should be considered in practice cycle analysis. The results obtained in the present study are of importance to provide a good guidance for the performance evaluation and improvement of practical Diesel engines.
\end{abstract}

PACS: 05.70.Ln, 82.60.Fa, 88.05.-b

\section{Introduction}

The Diesel cycle is similar to the Otto cycle. The main difference between a Diesel cycle and an Otto cycle is in the process of heat addition. In other words, in the Diesel cycle, the heat addition occurs at constant pressure whereas in the Otto cycle it is at a constant volume. Accordingly, the Diesel cycle is often referred to as the constant-pressure cycle. The Diesel or compression ignition (CI) engines operate with much higher compression ratio than the Otto or spark ignition (SI) engines and thus have higher efficiencies.

To make the analysis of the engine cycle much more manageable, air standard cycles are used to describe the major processes occurring in internal combustion engines. Air is assumed to behave as an ideal gas, and all processes are considered to be reversible $[1,2]$. In practice, air standard analysis is useful for illustrating the thermodynamic aspects of an engine operation cycle.

For an ideal engine cycle, the heat losses do not occur, however, for a real engine cycle, the heat losses indeed exist and should not be negligible. It is recognized that heat loss strongly affects the overall performance of the internal combustion engine. If it is neglected, the analy-

\footnotetext{
* corresponding author; e-mail: sshou@mail.ksu.edu.tw
}

sis will just depend on the ideal air standard cycle. Some attention has been paid to analyze the effects of heat transfer losses on the performance of internal combustion engines [3-7]. Klein [3] examined the effect of heat transfer through a cylinder wall on work output of the Otto and Diesel cycles. Chen et al. $[4,5]$ derived the relations between net power output and the efficiency of the Diesel and Otto cycles with considerations of heat loss through the cylinder wall. Hou [6] studied the effect of heat loss through a cylinder wall on performance of the dual cycle. Moreover, Hou [7] made comparison of performance of air-standard Atkinson and Otto cycles with heat transfer considerations.

In addition to heat loss, friction has a significant effect on the performance but it is omitted in ideal engine cycles. Taking into account the friction loss of the piston, Angulo-Brown et al. [8], Chen et al. [9] and Wang et al. [10] modeled Otto, Diesel and dual cycles with friction-like loss, respectively. Furthermore, Chen et al. $[11,12]$ and Ge et al. [13] derived the characteristics of power and efficiency for Otto, Dual and Miller cycles with considerations of heat transfer and friction-like term losses. The above studies [3-13] were done without considering the variable specific heats of working fluid. However, in the real engine cycle, the specific heat of working fluid is not a constant and should be considered in practical cycle analysis [14-18]. 
In those studies [3-18], the heat addition process for an air standard cycle has been widely described as subtraction from the fuel's chemical energy of an arbitrary heat loss parameter times the average temperature of the heat adding period. That is, the heat transfer to the cylinder walls is assumed to be a linear function of the difference between the average gas and cylinder wall temperatures during the energy release process. However, the heat leakage parameter and the fuel's energy depend on each other. Their valid ranges given in the literature affect the feasibility of air standard cycles. If they are selected arbitrarily, they will present unrealistic results and make the air standard cycles unfeasible [19]. For this reason, a more realistic and precise relationship between the fuel's chemical energy and the heat leakage needs to be derived through the resulting temperature [19]. Therefore, the performance analysis of any internal combustion engine can be covered by a more realistic and valid range of the heat loss parameter and the fuel's energy.

However, Ozsoysal's study [19] was only focused on the temperature limitations and no performance analysis was presented. Moreover, his study was done without considering the effects of variable specific heats of working fluid and friction. In particular, no performance analysis is available in the literature with emphasis on the Diesel cycle with considerations of variable specific heats of working fluid, friction and heat leakage characterized by a percentage of fuel's energy.

The purpose of this study is to explore these effects (i.e., variable specific heats of working fluid, friction and heat loss characterized by a percentage of fuel's energy) on the net work output and the indicated thermal efficiency of an air standard Diesel cycle. In the present study, we relax the assumptions that there are no heat losses during combustion, that there are no friction losses of the piston for the cycle, and that specific heats of working fluid are constant. In other words, heat transfer between the working fluid and the environment through the cylinder wall is considered and characterized by a percentage of fuel's energy; friction loss of the piston on the power output is taken into account. Furthermore, we consider the variable specific heats of working fluid that is significant in practical cycle analysis. The results obtained in the study may offer good guidance for the design and operation of the Diesel-cycle engine.

\section{Theoretical model}

Figure 1 shows the limitation of the maximum cycle temperature due to heat leakage in temperature-entropy diagram of an air standard Diesel cycle model. Thermodynamic cycle $1-2-3^{\prime}-4^{\prime}-1$ denotes the air standard Diesel cycle without heat leakage, while cycle 1-2-34-1 designates the air standard Diesel cycle with heat leakage. Process 1-2 is an isentropic compression from bottom-dead-center (BDC) to top-dead-center (TDC). The heat addition takes place in process $2-3$, which is isobatic. The isentropic expansion process, $3-4$, is the power or expansion stroke. The cycle is completed by an isochoric heat rejection process, $4-1$. The heat added to the working fluid per unit mass is due to combustion. The temperature at the completion of constant-pressure combustion $\left(T_{3}\right)$ depends on the heat input due to combustion and heat leakage through the cylinder wall. In this study, the amount of heat leakage is considered to be a percentage of the delivered fuel's energy [19]. The fuel's energy then is the sum of the actual fuel energy transferred to the working fluid and the heat leakage through the cylinder walls. If any heat leakage occurs, the maximum cycle temperature $\left(T_{3}\right)$ remains smaller than that of no heat leakage case $\left(T_{3^{\prime}}\right)$. When the total energy of the fuel is utilized, the maximum cycle temperature reaches undesirably high levels with regard to structural integrity. Hence, engine designers intend to restrict the maximum cycle temperature. Assuming that the heat engine is operated at the rate of $N$ cycles per second, then the total energy of the fuel per second input into the engine can be given by

$$
Q_{\text {fuel }}=N m_{\mathrm{f}} Q_{\mathrm{LHV}},
$$

and then the heat leakage per second is

$$
Q_{\text {leak }}=\alpha Q_{\text {fuel }}=\alpha N m_{\mathrm{f}} Q_{\mathrm{LHV}},
$$

where $m_{\mathrm{f}}$ is the delivered fuel mass into the cylinder, $Q_{\mathrm{LHV}}$ is the lower heating value of the fuel and $\alpha$ is an unknown percentage parameter having a value between 0 and 1.

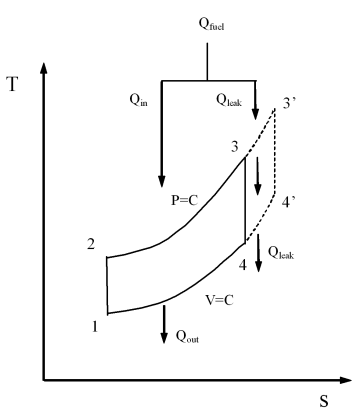

Fig. 1. $T-S$ diagram of an air standard Diesel cycle model.

Since the total energy of the delivered fuel $Q_{\text {fuel }}$ is assumed to be the sum of the heat added to the working fluid $Q_{\text {in }}$ and the heat leakage $Q_{\text {leak }}$, then

$$
Q_{\text {in }}=Q_{\text {fuel }}-Q_{\text {leak }}=(1-\alpha) N m_{\mathrm{f}} Q_{\text {LHV }} .
$$

In practical internal combustion engine cycle, constant-pressure and constant-volume specific heats of the working fluid are variable and these variations will greatly affect the performance of the cycle. According to Ref. [14], it can be assumed that the specific heats of the working fluid are functions of temperature alone and have the following linear forms:

$$
C_{p m}=a_{p}+k_{1} T
$$


and

$$
C_{v m}=b_{v}+k_{1} T,
$$

where $C_{p m}$ and $C_{v m}$ are, respectively, the specific heats with respect to constant pressure and volume. $a_{p}, b_{v}$ and $k_{1}$ are constants. Accordingly, the gas constant $(R)$ of the working fluid can be expressed as

$$
R=C_{p m}-C_{v m}=a_{p}-b_{v} .
$$

The temperature is restricted as the maximum temperature in the cycle as $T_{3}$, the available energy $Q_{\text {in }}$ during the heat addition per second can be written as

$$
\begin{aligned}
& Q_{\text {in }}=N m_{\mathrm{a}} \int_{T_{2}}^{T_{3}} C_{p m} \mathrm{~d} T \\
& \quad=N m_{\mathrm{a}} \int_{T_{2}}^{T_{3}}\left(a_{p}+k_{1} T\right) \mathrm{d} T=N m_{\mathrm{a}}\left[a_{p}\left(T_{3}-T_{2}\right)\right. \\
& \left.\quad+0.5 k_{1}\left(T_{3}^{2}-T_{2}^{2}\right)\right] .
\end{aligned}
$$

Combining Eqs. (3) and (7) yields

$$
\begin{aligned}
& N m_{\mathrm{a}}\left[a_{p}\left(T_{3}-T_{2}\right)+0.5 k_{1}\left(T_{3}^{2}-T_{2}^{2}\right)\right] \\
& \quad=(1-\alpha) N m_{\mathrm{f}} Q_{\mathrm{LHV}} .
\end{aligned}
$$

Dividing Eq. (8) by the amount of air mass $m_{\mathrm{a}}$, then we have

$$
\alpha=1-\frac{\lambda\left(m_{\mathrm{a}} / m_{\mathrm{f}}\right)_{\mathrm{s}}}{Q_{\mathrm{LHV}}}\left[a_{p}\left(T_{3}-T_{2}\right)+0.5 k_{1}\left(T_{3}^{2}-T_{2}^{2}\right)\right],
$$

Or

$$
\begin{aligned}
& T_{2}=\left\{-a_{p}+\right. \\
& \left.\sqrt{a_{p}^{2}+2 k_{1}\left[0.5 k_{1} T_{3}^{2}+a_{p} T_{3}-(1-\alpha) \frac{Q_{\mathrm{LHV}}}{\lambda\left(m_{\mathrm{a}} / m_{\mathrm{f}}\right)_{\mathrm{s}}}\right]}\right\} / k_{1},
\end{aligned}
$$

where $\lambda$ is the excess air coefficient defined as $\lambda=$ $\left(m_{\mathrm{a}} / m_{\mathrm{f}}\right) /\left(m_{\mathrm{a}} / m_{\mathrm{f}}\right)_{\mathrm{s}}\left(m_{\mathrm{a}} / m_{\mathrm{f}}\right)$ is air-fuel ratio and the subscripts a, f, and s, respectively, denote air, fuel and the stoichiometric condition.

The first condition for realizing a feasible cycle is $T_{2} \leq$ $T_{3}\left(=T_{\max }\right)$, so that

$$
\alpha \leq 1 \text {. }
$$

The upper limit for the percentage of heat leakage is then found as $\alpha_{\max }=1$. The second condition, $T_{2} \geq$ $T_{1}\left(=T_{\min }\right)$, is utilized to determine the lower limit as follows:

$$
\alpha \geq 1-\frac{\lambda\left(m_{\mathrm{a}} / m_{\mathrm{f}}\right)_{\mathrm{s}}}{2 Q_{\mathrm{LHV}}}\left[k_{1}\left(T_{3}^{2}-T_{1}^{2}\right)+2 a_{p}\left(T_{3}-T_{1}\right)\right] .
$$

Hence, the minimum value of $\alpha$ is expressed as

$$
\alpha_{\min }=1-\frac{\lambda\left(m_{\mathrm{a}} / m_{\mathrm{f}}\right)_{\mathrm{s}}}{2 Q_{\mathrm{LHV}}}\left[k_{1}\left(T_{3}^{2}-T_{1}^{2}\right)+2 a_{p}\left(T_{3}-T_{1}\right)\right] .
$$

The heat rejected per second by the working fluid $\left(Q_{\text {out }}\right)$ during process $4 \rightarrow 1$ is

$$
\begin{gathered}
Q_{\text {out }}=N m_{\mathrm{a}} \int_{T_{1}}^{T_{4}} C_{v m} \mathrm{~d} T=N m_{\mathrm{a}} \int_{T_{1}}^{T_{4}}\left(b_{v}+k_{1} T\right) \mathrm{d} T \\
=N m_{\mathrm{a}}\left[b_{v}\left(T_{4}-T_{1}\right)+0.5 k_{1}\left(T_{4}^{2}-T_{1}^{2}\right)\right] .
\end{gathered}
$$

The adiabatic exponent $k=C_{p m} / C_{v m}$ will vary with temperature since both $C_{p m}$ and $C_{v m}$ are dependent on temperature. Accordingly, the equation often used in reversible adiabatic process with constant $k$ cannot be used in reversible adiabatic process with variable $k$. According to Ref. [14], however, a suitable engineering approximation for reversible adiabatic process with variable $k$ can be made, i.e., this process can be divided into infinitesimally small processes, for each of these processes, adiabatic exponent $k$ can be regarded as constant. For instance, for any reversible adiabatic process between states $i$ and $j$, we can regard the process as that it consists of numerous infinitesimally small processes with constant $k$. For any of these processes, when small changes in temperature $\mathrm{d} T$, and in volume $\mathrm{d} V$ of the working fluid take place, the equation for reversible adiabatic process with variable $k$ can be written as follows:

$$
T V^{k-1}=(T+\mathrm{d} T)(V+\mathrm{d} V)^{k-1} .
$$

From Eq. (15), we get the following equation:

$$
k_{1}\left(T_{j}-T_{i}\right)+b_{v} \ln \left(T_{j} / T_{i}\right)=-R \ln \left(V_{j} / V_{i}\right) .
$$

The compression ratio $\left(\gamma_{\mathrm{c}}\right)$ is defined as $\gamma_{\mathrm{c}}=V_{1} / V_{2}$. Therefore, the equations for processes $1 \rightarrow 2$ and $3 \rightarrow 4$ are shown, respectively, by the following equations:

$$
k_{1}\left(T_{2}-T_{1}\right)+b_{v} \ln \left(T_{2} / T_{1}\right)=R \ln \gamma_{\mathrm{c}},
$$
and

$$
k_{1}\left(T_{3}-T_{4}\right)+b_{v} \ln \left(T_{3} / T_{4}\right)=R \ln \left(T_{2} \gamma_{\mathrm{c}} / T_{3}\right) .
$$

The power output without friction losses is given by

$$
\begin{aligned}
& P_{\mathrm{R}}=Q_{\text {in }}-Q_{\text {out }}=N m_{\mathrm{a}}\left[a_{p}\left(T_{3}-T_{2}\right)-b_{v}\left(T_{4}-T_{1}\right)\right. \\
& \left.\quad+0.5 k_{1}\left(T_{3}^{2}+T_{1}^{2}-T_{2}^{2}-T_{4}^{2}\right)\right] .
\end{aligned}
$$

Considering the friction of the piston and assuming a dissipation term represented by a friction force $\left(f_{\mu}\right)$ which is linearly proportional to the velocity of the piston [8-10], then we have

$$
f_{\mu}=-\mu v=-\mu \frac{\mathrm{d} x}{\mathrm{~d} t},
$$

where $\mu$ is the coefficient of friction, which takes into account the global losses on the power output, $x$ is the piston's displacement and $v$ is the piston's velocity. Therefore, the power lost due to friction is

$$
P_{\mu}=f_{\mu} v=-\mu\left(\frac{\mathrm{d} x}{\mathrm{~d} t}\right)^{2}=-\mu v^{2} .
$$

For a four stroke cycle engine, the total distance the piston travels per cycle is

$$
\begin{aligned}
& 4 L=4\left(x_{1}-x_{2}\right)=4 x_{2}\left(x_{1} / x_{2}-1\right) \\
& \quad=4 x_{2}\left(\gamma_{\mathrm{c}}-1\right),
\end{aligned}
$$

where $x_{1}$ and $x_{2}$ are the piston's position corresponding 
to the maximum and minimum volume, respectively. $L$ is the stroke of the piston.

Running at $N$ cycles per second, the mean velocity of the piston is

$$
\bar{v}=4 L N .
$$

Therefore, the net actual power output of the Diesel-cycle engine can be written as

$$
\begin{aligned}
P & =P_{\mathrm{R}}-\left|P_{\mu}\right|=N m_{\mathrm{a}}\left[a_{p}\left(T_{3}-T_{2}\right)-b_{v}\left(T_{4}-T_{1}\right)\right. \\
& \left.+0.5 k_{1}\left(T_{3}^{2}+T_{1}^{2}-T_{2}^{2}-T_{4}^{2}\right)\right] \\
& -16 \mu\left[N x_{2}\left(\gamma_{\mathrm{c}}-1\right)\right]^{2} .
\end{aligned}
$$

Also, the efficiency of the Diesel-cycle engine can be expressed by

$$
\begin{aligned}
\eta & =\frac{P}{Q_{\text {in }}}=\left\{m _ { \mathrm { a } } \left[a_{p}\left(T_{3}-T_{2}\right)-b_{v}\left(T_{4}-T_{1}\right)\right.\right. \\
& \left.+0.5 k_{1}\left(T_{3}^{2}+T_{1}^{2}-T_{2}^{2}-T_{4}^{2}\right)\right] \\
& \left.-16 \mu N\left[x_{2}\left(\gamma_{\mathrm{c}}-1\right)\right]^{2}\right\} \\
& \times\left\{m_{\mathrm{a}}\left[a_{p}\left(T_{3}-T_{2}\right)+0.5 k_{1}\left(T_{3}^{2}-T_{2}^{2}\right)\right]\right\}^{-1} .
\end{aligned}
$$

When $T_{1}, T_{3}$ and $\gamma_{\mathrm{c}}$ are given, then $T_{2}$ can be obtained from Eq. (17) and $T_{4}$ can be found from Eq. (18). Finally, by substituting $T_{1}, T_{2}, T_{3}$ and $T_{4}$ into Eqs. (24) and (25), respectively, the power output and the efficiency of the Diesel-cycle engine can be obtained. Therefore, the relations between the power output, the efficiency and the compression ratio can be derived.

\section{Results and discussion}

According to Refs. [14, 17, 20], the following constants and ranges of parameters are used in the calculations: $b_{v}=0.6858-0.8239 \mathrm{~kJ} /(\mathrm{kg} \mathrm{K}), m_{\mathrm{a}}=1.26 \times 10^{-3} \mathrm{~kg}, T_{1}=$ $\left.300-400 \mathrm{~K}, k_{1}=0.000133-0.00034 \mathrm{~kJ} /(\mathrm{kg} \mathrm{K})^{2}\right), x_{2}=$ $0.01 \mathrm{~m}, N=30, Q_{\mathrm{LHV}}=43200 \mathrm{~kJ} / \mathrm{kg}$ and $\mu=0.0129$ $0.0169 \mathrm{kN} \mathrm{s} / \mathrm{m}$. This study focuses on the limitation of maximum cycle temperature $T_{3}$ instead of $T_{3^{\prime}}$ due to the varying heat leakage conditions. Numerical examples are shown as follows.

Figure 2 shows the variation of the heat leakage percentage $(\alpha)$ with respect to the maximum cycle temperature $\left(T_{3}\right)$ and the volumetric compression ratio $\left(\gamma_{\mathrm{c}}\right)$. It can be found that the maximum cycle temperature plays a dominant role on the quantity of heat leakage. For a fixed compression ratio, the larger maximum cycle temperature can be obtained as the heat leakage percentage parameter is smaller. To achieve a fixed cycle maximum temperature, the heat leakage percentage parameter increases with the increase of compression ratio. The most important point here is that some values of the heat leakage percentage might be insufficient for a feasible Diesel cycle. Therefore, acceptable values for $\alpha$ could only be achieved from the definition of $\alpha_{\min }$ given by Eq. (13).

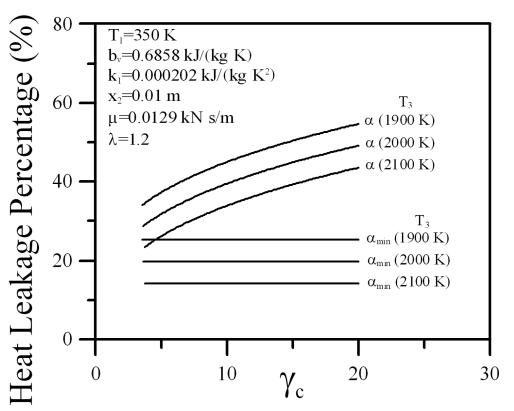

Fig. 2. The variation of the heat leakage percentage $(\alpha)$ with respect to the maximum cycle temperature $\left(T_{3}\right)$ and the volumetric compression ratio $\left(\gamma_{\mathrm{c}}\right)$.

Figure 3 illustrates the variation of the heat leakage percentage $(\alpha)$ with respect to the air-fuel ratio $\left(m_{\mathrm{a}} / m_{\mathrm{f}}\right)$ or the excess air coefficient $(\lambda)$ and the volumetric compression ratio $\left(\gamma_{c}\right)$. The results show that the excess air coefficient also plays an important role on the quantity of heat leakage. To obtain a fixed maximum cycle temperature $\left(T_{3}=2000 \mathrm{~K}\right)$, the heat leakage percentage parameter increases with the increase of compression ratio. For fixed maximum cycle temperature and compression ratio, heat leakage percentage parameter decreases with increase of the excess air coefficient. Similarly to Fig. 2, suitable values for $\alpha$ could only be achieved from the definition of $\alpha_{\min }$ given by Eq. (13).

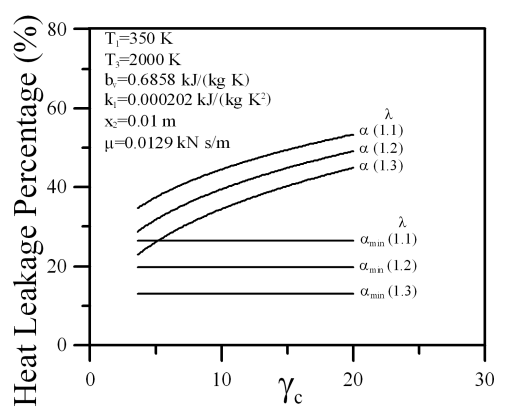

Fig. 3. The variation of the heat leakage percentage $(\alpha)$ with respect to the air-fuel ratio $\left(m_{\mathrm{a}} / m_{\mathrm{f}}\right)$ or the excess air coefficient $(\lambda)$ and the volumetric compression ratio $\left(\gamma_{\mathrm{c}}\right)$.

Figure 4 depicts the influence of maximum cycle temperature $\left(T_{3}\right)$ on the cycle performance. The power output given by Eq. (24) is a convex function with a single maximum for the optimum compression ratio, as shown in Fig. 4a. Increase in compression ratio first leads to an increase in power output, and after reaching a peak, then the net power output decreases dramatically with further increase in compression ratio. The behavior of the efficiency versus compression ratio plot is similar to that for the power output, as shown in Fig. 4b. Additionally, Fig. 4 illustrates that increasing $T_{3}$ corresponds to enlarging the amount of heat added to the engine due to 
combustion, and, therefore, $T_{3}$ has a positive effect on the $P-\gamma_{\mathrm{c}}$ and $\eta-\gamma_{\mathrm{c}}$ characteristic curves. In other words, for a given $\gamma_{\mathrm{c}}$ the power output and efficiency increase with increasing $T_{3}$ and the maximum power output and its corresponding efficiency increase with the increase of $T_{3}$. Furthermore, it can be seen that the values of $\gamma_{c}$ at the maximum power output or at the maximum efficiency increase with the increase of $T_{3}$.

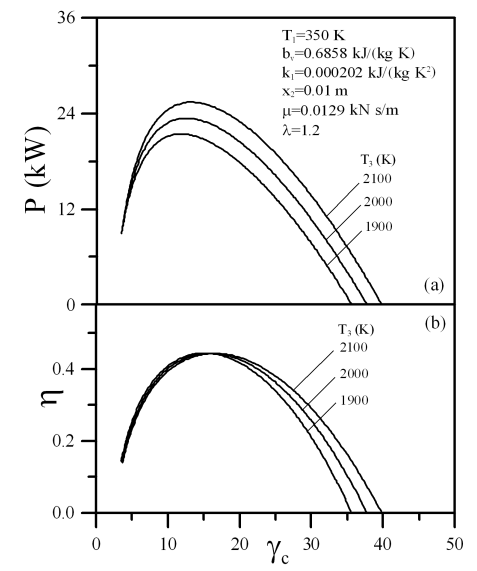

Fig. 4. (a) The influence of maximum cycle temperature $\left(T_{3}\right)$ on the variation of power output $(P)$ with compression ratio $\left(\gamma_{\mathrm{c}}\right)$; (b) the influence of maximum cycle temperature $\left(T_{3}\right)$ on the variation of efficiency $(\eta)$ with compression ratio $\left(\gamma_{c}\right)$.

It has been reported that for a real heat engine, the maximum power and maximum efficiency operating points are usually relatively close [20]. This is reflected through loop-shaped power versus efficiency plots. As is shown in Fig. 5, we also obtain the loop-shaped power output versus efficiency curves which reflect the performance characteristics of a real irreversible Diesel-cycle engine. It can be observed that the maximum power output, the maximum efficiency, the power at maximum efficiency and the efficiency at maximum power will increase with the increase of $T_{3}$.

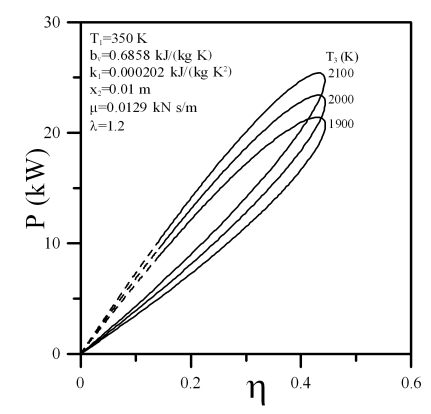

Fig. 5. The influence of maximum cycle temperature $\left(T_{3}\right)$ on the power output $(P)$ versus efficiency $(\eta)$ characteristic curves.

Figures 6 and 7 show the influence of the parameter $b_{v}$ related to the variable specific heats of the working fluid

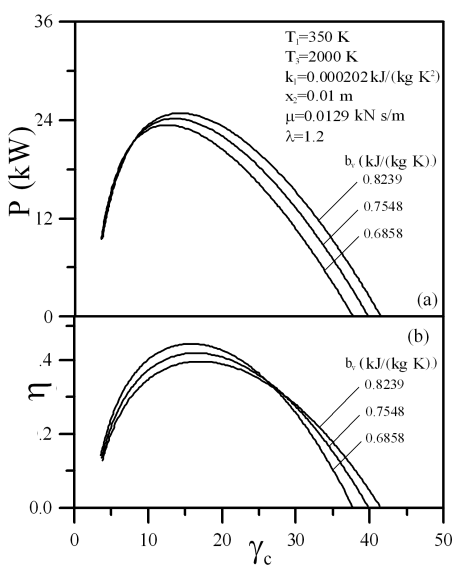

Fig. 6. (a) The influence of $b_{v}$ on the variation of power output $(P)$ with compression ratio $\left(\gamma_{\mathrm{c}}\right)$; (b) the influence of $b_{v}$ on the variation of efficiency $(\eta)$ with compression ratio $\left(\gamma_{\mathrm{c}}\right)$.

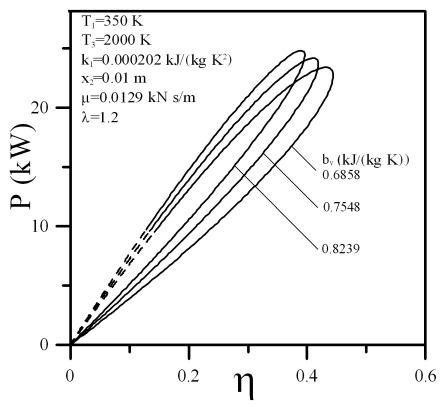

Fig. 7. The influence of $b_{v}$ on the power output $(P)$ versus efficiency $(\eta)$ characteristic curves.

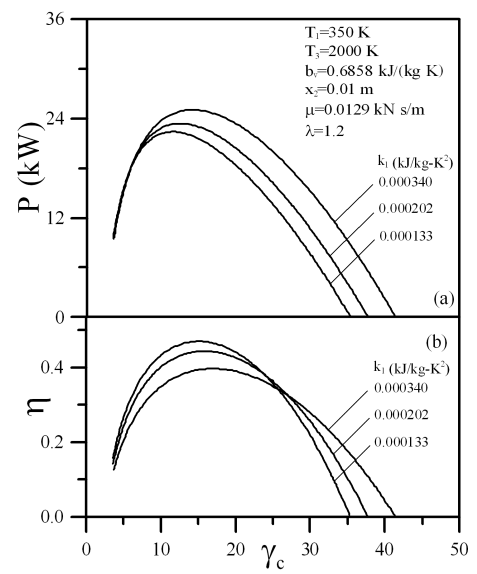

Fig. 8. (a) The influence of $k_{1}$ on the variation of power output $(P)$ with compression ratio $\left(\gamma_{\mathrm{c}}\right)$; (b) the influence of $k_{1}$ on the variation of efficiency $(\eta)$ with compression ratio $\left(\gamma_{\mathrm{c}}\right)$. 


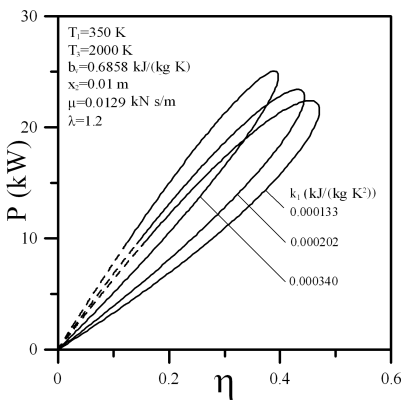

Fig. 9. The influence of $k_{1}$ on the power output $(P)$ versus efficiency $(\eta)$ characteristic curves.

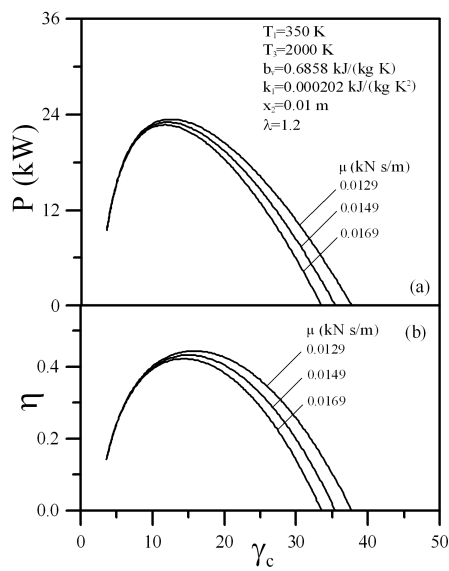

Fig. 10. (a) The influence of $\mu$ on the variation of power output $(P)$ with compression ratio $\left(\gamma_{\mathrm{c}}\right)$; $(\mathrm{b})$ the influence of $\mu$ on the variation of efficiency $(\eta)$ with compression ratio $\left(\gamma_{\mathrm{c}}\right)$.

on the performance of the Diesel cycle. For a fixed $k_{1}$, a larger $b_{v}$ corresponds to a greater value of the specific heat with constant volume $\left(C_{v m}\right)$ or the specific heat with constant pressure $\left(C_{p m}\right)$. Figure 6 a demonstrates that for a given $\gamma_{\mathrm{c}}$, the maximum power output and the working range of the cycle increase with the increase of $b_{v}$, nevertheless, Fig. $6 \mathrm{~b}$ shows that the maximum efficiency decreases with the increase of $b_{v}$. It is noted that

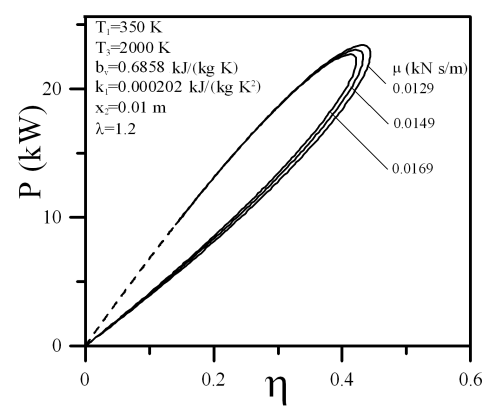

Fig. 11. The influence of $\mu$ on the power output $(P)$ versus efficiency $(\eta)$ characteristic curves.

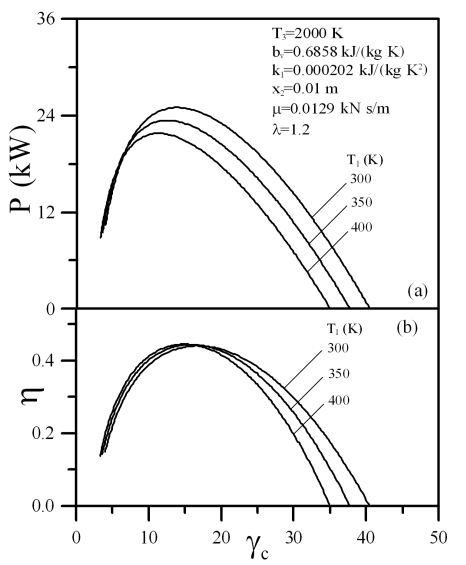

Fig. 12. (a) The influence of intake temperature $\left(T_{1}\right)$ on the variation of power output $(P)$ with compression ratio $\left(\gamma_{\mathrm{c}}\right)$; (b) the influence of intake temperature $\left(T_{1}\right)$ on the variation of efficiency $(\eta)$ with compression ratio $\left(\gamma_{\mathrm{c}}\right)$.

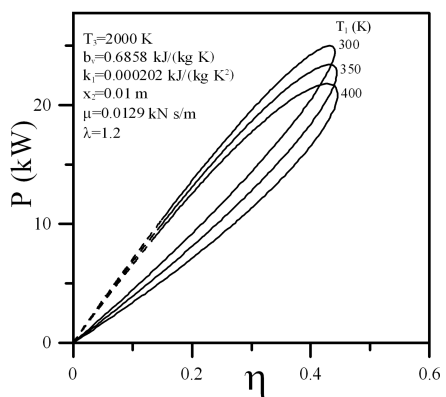

Fig. 13. The influence of intake temperature $\left(T_{1}\right)$ on the power output $(P)$ versus efficiency $(\eta)$ characteristic curves.

the parameter $b_{v}$ has an important influence on the compression ratio where the maximum power or efficiency occurs. The values of $\gamma_{c}$ at the maximum power output or at the maximum efficiency increase with the increase of $b_{v}$, as shown in Fig. 6. It can be found from Fig. 7 that the curves of power output versus efficiency are also loop-shaped. It shows that, with the increase of $b_{v}$, the maximum power output and the power at maximum efficiency increase, while the maximum efficiency and the efficiency at maximum power output decrease.

Figures 8 and 9 depict the influence of the parameter $k_{1}$ related to the variable specific heats of the working fluid on the performance of the Diesel cycle. For a given $b_{v}$, a larger $k_{1}$ corresponds to a greater value of the specific heats with constant volume $\left(C_{v m}\right)$ or the specific heat with constant pressure $\left(C_{p m}\right)$. Figure 8 shows that $k_{1}$ has the same influence as $b_{v}$ (shown in Fig. 6) on the performance of the cycle. That is, for a given $\gamma_{c}$ the power output and the working range of the cycle increase with increasing $k_{1}$, as shown in Fig. 8a, while the efficiency decreases with the increase of $k_{1}$, as depicted in Fig. 8b. 
Also, it can be found that the parameter $k_{1}$ has a significant influence on the loop-shaped curves for the power output versus efficiency plots. With the increase of $k_{1}$, the maximum power output and the power at maximum efficiency increase, while the maximum efficiency and the efficiency at maximum power output decrease, as shown in Fig. 9.

Figures 10 and 11 show the influence of the friction-like term loss $(\mu)$ on the performance of the Diesel cycle. It is clear that the parameter $\mu$ has a negative effect on the performance. As shown in Fig. 10, the maximum power output, the maximum efficiency and the value of the compression ratio at maximum power output or at maximum efficiency will decrease with increasing $\mu$. Moreover, Fig. 11 shows that the maximum power output, the maximum efficiency, the power at maximum efficiency and the efficiency at maximum power will decrease with the increase of $\mu$.

Figures 12 and 13 demonstrate the influence of intake temperature $\left(T_{1}\right)$ on the performance of the Diesel cycle. It can be found from Fig. 12 that for a restricted maximum cycle temperature $T_{3}=2000 \mathrm{~K}$, the maximum power output, the maximum efficiency, the compression ratio at maximum power output and the compression ratio at maximum efficiency of the Diesel cycle decrease with the increase of $T_{1}$. Loop-shaped curves of power versus efficiency plots are also shown in Fig. 13. It also reveals that as $T_{1}$ increases, the maximum power output, the maximum efficiency, the efficiency at maximum power output and the power output at maximum efficiency decrease.

\section{Conclusions}

The influences of heat loss as a percentage of fuel's energy, friction and variable specific heats of working fluid on the performance of a Diesel engine under the restriction of maximum cycle temperature are presented in this study. The results are summarized as follows.

(1) The maximum power output, the maximum efficiency, the power at maximum efficiency, the efficiency at maximum power and the value of the compression ratio when the power output or the efficiency is maximum increase with the increase of maximum cycle temperature $T_{3}$.

(2) The parameters $b_{v}$ and $k_{1}$ related to the variable specific heats of the working fluid have a significant influence on the performance of the Diesel cycle. For a fixed $k_{1}\left(\right.$ or $\left.b_{v}\right)$, a larger $b_{v}\left(\right.$ or $\left.k_{1}\right)$ corresponds to a greater value of the specific heats with constant volume $\left(C_{v m}\right)$. For a given compression ratio $\gamma_{c}$, the power output and the working range of the cycle increase with the increase of the parameter $b_{v}$ or $k_{1}$, nevertheless, the efficiency decreases with the increase of $b_{v}$ or $k_{1}$. Furthermore, with the increase of $b_{v}$, the maximum power output and the power at maximum efficiency increase, while the maximum efficiency and the efficiency at maximum power output decrease.

(3) The influence of the friction-like term loss $\mu$ has a negative effect on the performance. Therefore, the maximum power output, the maximum efficiency, the power at maximum efficiency, and the efficiency at maximum power will decrease with the increase of $\mu$.

(4) The maximum power output, the maximum efficiency, the compression ratio at maximum power output, and the compression ratio at maximum efficiency of the Diesel cycle decrease with the increase of intake temperature $T_{1}$. The efficiency at maximum power output and the power output at maximum efficiency decrease with increasing $T_{1}$.

(5) It is noteworthy that the effects of heat loss as a percentage of fuel's energy and friction loss on the performance of a Diesel-cycle engine with considerations of variable specific heats of working fluid are significant and should be considered in practical cycle analysis. The results obtained in the present study are of importance to provide a good guidance for the performance evaluation and improvement of practical Diesel-cycle engines.

\section{Acknowledgments}

This work was supported by the National Science Council, Taiwan, ROC, under contract NSC95-2212-E$-168-006$.

\section{References}

[1] W.W. Pulkrabek, Engineering Fundamentals of the Internal Combustion Engine, Pearson Education Inc., Upper Saddle River, NJ 2002.

[2] J. B Heywood, Internal Combustion Engine Fundamentals, McGraw-Hill, New York 1997.

[3] S.A. Klein, Trans. ASME J. Eng. Gas Turbine Power 113, 511 (1991).

[4] L. Chen, F. Zen, F. Sun, C. Wu, Energy Int. J. 21, 1201 (1996).

[5] L. Chen, C. Wu, F. Sun, S. Cao, Energy Conv. Manag. 39, 643 (1998).

[6] S.S. Hou, Energy Conv. Manag. 45, 3003 (2004).

[7] S.S. Hou, Energy Conv. Manag. 48, 1683 (2007).

[8] F. Angulo-Brown, J. Fernandez-Betanzos, C.A. Diaz-Pico, Eur. J. Phys. 15, 38 (1994).

[9] L. Chen, J. Lin, J. Luo, F. Sun, C. Wu, Int. J. Energy Res. 26, 965 (2002).

[10] W. Wang, L. Chen, F. Sun, C. Wu, Exergy An Int. J. 2, 340 (2002).

[11] L. Chen, T. Zheng, F. Sun, C. Wu, Int. J. Ambient Energy 24, 195 (2003).

[12] L. Chen, F. Sun, C. Wu, Appl. Energy 79, 3 (2004). 
[13] Y. Ge, L. Chen, F. Sun, Int. Commun. Heat Mass Transfer 32, 1045 (2005).

[14] Y. Ge, L. Chen, F. Sun, C. Wu, Int. J. Therm. Sci. 44, 506 (2005).

[15] A. Al-Sarkhi, J.O. Jaber, M. Abu-Qudais, S.D. Probert, Appl. Energy 83, 153 (2006).

[16] A. Al-Sarkhi, J.O. Jaber, M. Abu-Qudais, S.D. Probert, Appl. Energy 83, 343 (2006).

[17] Y. Ge, L. Chen, F. Sun, C. Wu, Appl. Energy 83, $1210(2006)$
[18] R. Ebrahimi, Acta Phys. Pol. A 117, 887 (2010).

[19] O.A. Ozsoysal, Energy Conv. Manag. 47, 1051 (2006).

[20] M. Mozurkewich, R.S. Berry, J. Appl. Phys. 53, 34 (1982). 